\title{
Restoring a Radionuclide Contaminated Aquatic Ecosystem: A Group Decision Making Problem with Incomplete Information within MAUT Accounting for Veto
}

\author{
Antonio Jiménez-Martín ${ }^{1}$ - Eduardo Gallego ${ }^{2} \cdot$ Alfonso Mateos $^{1}$ • Juan A. \\ Fernández del Pozo ${ }^{1}$
}

\begin{abstract}
In this paper we consider the evaluation of intervention countermeasures for the restoration of a radionuclide contaminated aquatic ecosystem, simultaneously taking into account several conflicting objectives, like environmental, social and economic impacts. We propose an extension of the additive multi-attribute utility model to incorporate the concept of veto to deal with this group decision-making problem. Moreover, we consider what is known as decision-making with partial, imprecise or incomplete information, which accounts for uncertainty about the alternative performances and imprecision concerning DM preferences by means of intervals or ordinal information. Veto values for the most important DMs are used to define veto ranges, whereas veto values corresponding to the other less important DMs are partially taken into account, leading to the construction of adjust ranges. We then build the veto and adjust functions into the additive model, and a dominance matrix accounting for incomplete information is computed. A dominance measuring method is then used to derive a ranking of alternatives for each DM, which are then aggregated taking into account their relative importance.
\end{abstract}

Antonio Jiménez-Martín antonio.jimenez@upm.es http:/www.upm.es

Eduardo Gallego eduardo.gallego@upm.es

Alfonso Mateos

alfonso.mateos@upm.es

Juan A. Fernández del Pozo

juan.fdezpozo.salamanca@upm.es

1 E.T.S.I. Informáticos, Universidad Politécnica de Madrid, 28660 Boadilla del Monte, Spain

2 E.T.S.I. Industriales, Universidad Politécnica de Madrid, 28006 Madrid, Spain 


\section{Introduction}

The additive model is considered to be a valid approach within multi-attribute value/utility theory (MAVT/MAUT) in most practical situations for the reasons described in Raiffa (1982), Stewart (1996).

However, the information available in most real complex decision-making problems is not precise. Inputs are often described within prescribed bounds or as just satisfying certain relations. Different authors refer to this situation as decision-making with imprecise information, incomplete information or partial information (Ríos Insua 1990; Ríos and French 1991).

Several reasons are given in the literature that justify why a decision-maker (DM) may wish to provide imprecise information (Sarabando and Dias 2010; Weber 1987). For example, performances that reflect social or environmental impacts may be intangible or non-monetary, and performances may be taken from statistics or measurements. Besides, a DM might prefer not to reveal his/her preferences in public or could feel more comfortable providing a scale to represent attribute importance.

Sarabando and Dias (2009) provided a brief overview of approaches proposed by different authors within the MAVT/MAUT framework to deal with imprecise information, including the modification of four classical decision rules to encompass an imprecise decision context on the basis of the absolute dominance notion (Puerto et al. 2000; Salo and Hämäläinen 2001), surrogate weighting methods (Barron and Barret 1996; Stillwell et al. 1981), in which a weight vector is selected from a set of admissible weights to represent the set, which is then used to evaluate the alternatives by means of the multi-attribute value model; stochastic multicriteria acceptability analysis(SMAA) (Lahdelma et al. 1998), SMAA-2 (Lahdelma and Salminen 2001) and SMAA-O (Lahdelma et al.2003), which are based on the idea of volume computations; or the assessment of dominance and potential optimality on the basis of the pairwise dominance notion (Eum et al. 2001; Lee et al. 2002; Mateos et al. 2007).

A more recent approach for dealing with imprecise information, which consists of computing different measures of dominance to derive a ranking of alternatives, are dominance measuring methods (DMMs) (Ahn and Park 2008; Aguayo 2014). DMMs are based on the computation of a dominance matrix, $D$, including pairwise dominance values, which are exploited in different ways to derive measures of dominance to rank the alternatives under consideration.

Besides, real complex decision-making problems usually take into account the preferences of a group of DMs. Many multi-attribute methods for individual decisionmaking have been extended to a group framework. Two different approaches for the aggregation process can be considered.

First, the aggregation process could be performed at the level of individual parameters of the decision model (component utilities and weights). For instance, Mateos et al. (2003) develop a decision support system based on Monte-Carlo simulation techniques, where the DMs' preferences are elicited separately, as a starting point 
for a negotiation process. Jiménez et al. (2005) propose an iterative process in which the imprecise sets of utilities and weights corresponding to the different DMs are successively tightened.

On the other hand, the aggregation process could be performed at the level of evaluations of the considered alternatives (Vetschera 1990). Different methods for aggregating rankings by different authors can be found in the literature. Lin (2010) discusses three classes of methods, namely distribution-based methods, for instance, the original Thurstone scaling and its extensions (Green 1978); heuristic methods, ranging from simple arithmetic averages of ranks (Bordas methods) Borda (1981) to Markov chains and stationary distributions (DeConde et al. 2006); and stochastic optimization search methods, such as the Kemeny optimal aggregation (Kemeny 1959).

Finally, the veto concept is considered as a real-world approach for representing the limits of DM preferences, and is thus an important tool in multicriteria and group decision-making. At the same time, this use of the veto concept is an attempt to account for the flawedness or ambiguity of the evaluation of alternatives in order to reach a consensus. The veto concept has been used both in non-compensatory and compensatory methods within multicriteria decision making (MCDM).

The concept of veto threshold could be related within MAUT to the definition of each attribute's preference bounds, whereby alternatives whose criteria are rated above or below these bounds are rejected by DMs depending on whether their utility function is increasing or decreasing and irrespective of the value that they take for other attributes (Sabio et al. 2015). Veto could be also applied to the rankings of alternatives derived by each DM (Daher and de Almeida 2012).

In this paper we consider a complex decision-making problem, the evaluation of intervention countermeasures for the restoration of a radionuclide contaminated aquatic ecosystem, simultaneously taking into account several conflicting objectives, like environmental, social and economic impacts.

We account for a decision-making within an imprecise information context, where the impacts of the countermeasures will be described under uncertainty and imprecise preferences of several experts will be incorporated into the analysis by means of ordinal information. Moreover, experts are allowed to provide vetoes expressed in terms of alternative performances.

We propose a methodology to deal with this group decision-making problem with imprecise information accounting for veto. The methodology consists of two aggregation phases. In the first phase, the additive multi-attribute model is first adapted to account for DM vetoes by adding a veto and an adjust function. A dominance measuring method accounting for the imprecise DM preferences is used to derive a ranking of alternatives for each DM. Note that although the respective DMs preferences concerning weights are considered for each ranking, the resulting ranking is influenced by the opinion of the other DMs by means of the veto and adjust functions.

In the second phase, the different rankings derived in the first phase are aggregated taking into account the relative importance of DMs to reach a group ranking using the order explicit algorithm method, an extension of Kemeny optimal aggregation.

The paper is structured as follows. Section 2 introduces the restoration of radionuclide contaminated aquatic ecosystems problem, which is structured by means of an 
objective hierarchy. Section 3 deals with group decision-making with incomplete information within MAUT. First, remedial countermeasures and their impacts in terms of the previously identified attributes are described under uncertainty by means of intervals or ordinal information. Then, DM preferences are imprecisely quantified, leading to classes of component utility functions and ordinal information about utilities/weights.

Section 4 introduces the origin of the veto concept and reviews how it was used in non-compensatory and compensatory methods within MCDM. Then, Sect. 4.1 describes an approach to deal with veto values in a group decision-making context. Section 5 proposes the methodology to deal with a group decision-making context with imprecise information accounting for veto on the basis of two aggregation phases. First, we introduce a dominance measuring method in Sect. 5.1 to derive a ranking of alternatives for each DM. We then analyze the aggregation of the resulting rankings taking into account the relative importance of DMs in Sect. 5.2. Section 6 discusses alternatives identified in the literature for incorporating veto values into the analysis, which are compared with our proposal. Finally, some conclusions are provided in Sect. 7 .

\section{Restoration of an Aquatic Ecosystem Contaminated by Radionuclides}

The evaluation of intervention countermeasures for the restoration of a radionuclide contaminated aquatic ecosystem was studied in depth as part of several European projects in which we participated: MOIRA (Monte et al. 2000), COMETES (Monte et al. 2002), EVANET-HYDRA (Monte et al. 2005), and EURANOS (Raskob et al. 2010). Throughout these projects, a synthetic, flexible and user-friendly computerized decision support system, MOIRA (Gallego et al. 2009; Monte et al. 2009), was implemented and tested on several real scenarios.

The source term in the scenario considered in this paper was postulated after a hypothetical severe accident at the Almaraz nuclear power plant (Spain). Taking into account the level-2 PSA (probabilistic safety analysis), the release category R6 was selected (Khatib-Rahbar et al. 1998), implying quite a sizeable and relatively credible release despite the extremely low probability of such a severe accident. Specifically, a release of $3 \%$ of the $\mathrm{Cs}^{137}$ and $0.062 \%$ of $\mathrm{Sr}^{90}$ inventories was considered.

For this particular scenario, we focus on a wetland area of Extremadura region, Lake Palancoso, which belongs to the Ejido Nuevo lake complex, located in the district of Campo Arañuelo. While not a source of drinking water, it has notable ecological value with a status of special protection area for birds. On top of that, the existence of recreational fishing, while not implying high collective doses due to the low total volume, could lead to unacceptable doses for the fishermen consuming their own captures. Furthermore, the area attracts a lot of tourists, due to fishing and birdwatching, some of the birds being in danger of extinction.

Lake Palancoso has a surface area of roughly $100,000 \mathrm{~m}^{2}$, whereas the catchment area is five times the size of the lake. Its depth is highly variable over the year since its volume is small, and it is situated at $270 \mathrm{~m}$ above sea level. Further information concerning chemical conditions at the lake, soil and weather conditions, land use, fish 


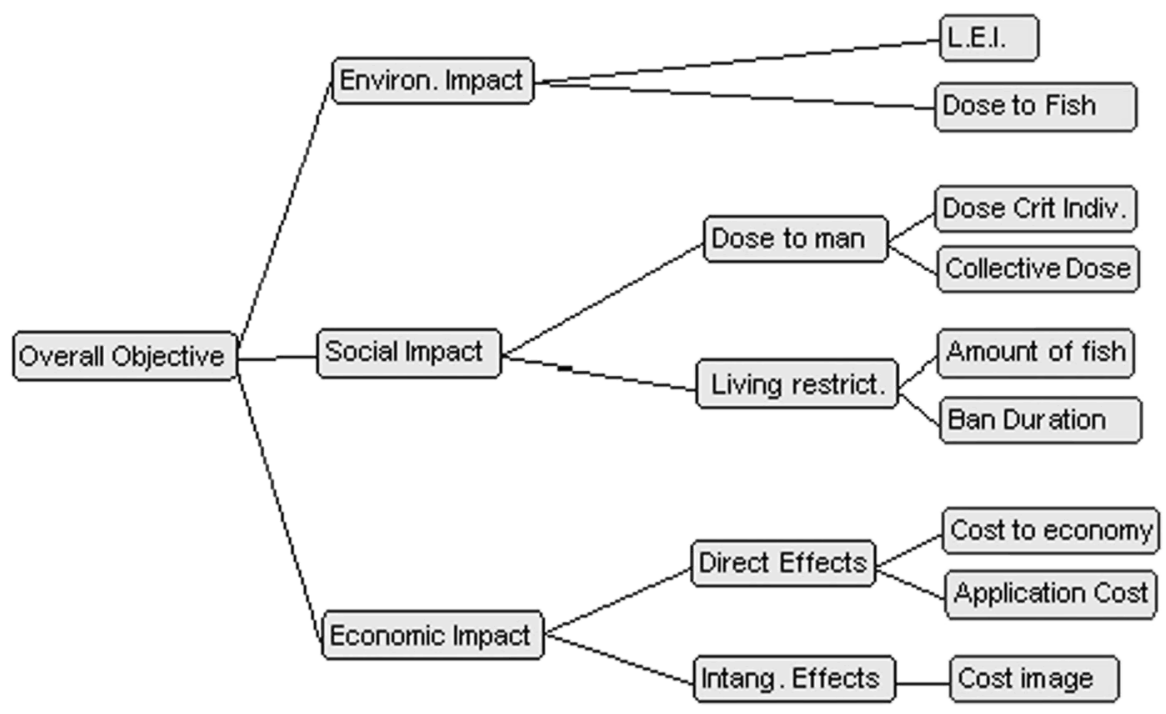

Fig. 1 Objective hierarchy

production and water withdrawal for human consumption is available in Gallego and Magán (2009).

RODOS (real-time on-line decision support for nuclear emergencies) (Ehrhardt et al. 1997) simulation was performed with the postulated release. Results showed that local contamination with $\mathrm{Cs}^{137}$ would be between $10^{4}$ and $10^{5} \mathrm{~Bq} / \mathrm{m}^{2}$. The conservative approach was taken and thus the maximum value was chosen. Besides, $\mathrm{Sr}^{90}$ fallout is around $1 / 30$ that of $\mathrm{Cs}^{137}$, i.e. $3.000 \mathrm{~Bq} / \mathrm{m}^{2}$.

\subsection{Structuring the Restoration Problem}

An objective hierarchy was built, including all the relevant aspects to be taken into account in the restoration problem, see Fig. 1.

Environmental Impact (Environ. Impact) is one of the main objectives of the decision analysis. It is divided into Lake Ecosystem Index (LEI) and Dose to Fish (Monte and Brittain 1998). LEI is a simple, rational approach for measuring the ecological status of a lake, by comparing normal values of key variables with their actual values and their influence on the fish yield, the phytoplankton biomass and the bottom fauna biomass (Håkanson et al. 2000).

Social Impact (Social Impact) is handled by two subobjectives: minimizing impact on health, Dose to Man, and Living Restrictions. Regarding dose to man, we focus on the Dose to Critical Individuals and Collective Dose. Dose to Critical Individuals refers to the effective dose received by individuals belonging to a critical group living in the area, drinking water and eating aquatic food and food grown on land irrigated with water from the contaminated body of water, whereas Collective Dose is a measure of the increased risk of serious latent health effects. 
Table 1 Attributes with continuous scale

\begin{tabular}{lll}
\hline & Unit & Range \\
\hline$X_{1}:$ Lake Ecosystem Index & LEI & {$[1,5]$} \\
$X_{3}:$ Dose Crit. Indiv. & $\mathrm{mSv}$ & {$[0,500]$} \\
$X_{4}:$ Collective Dose & $\mathrm{mSv} \times$ person & {$\left[0,12 \times 10^{4}\right]$} \\
$X_{5}:$ Amount of Fish & Tonnes & {$[0,100]$} \\
$X_{6}:$ Ban Duration & Months & {$[0,360]$} \\
$X_{7}:$ Costs to Economy & Euros & {$\left[0,10^{8}\right]$} \\
$X_{8}:$ Application Cost & Euros & {$\left[0,10^{7}\right]$} \\
\hline
\end{tabular}

As regards Living Restrictions, other impacts are taken into consideration. These include countermeasures affecting the direct consumption of fish for food or its processing in the food industry, drinking water and water used by the food industry, the use of water for crop irrigation and the recreational uses of water bodies. For all these objectives, the attributes will be the amount of fish affected by restrictions (Amount of Fish), as well as the duration of such restrictions (Ban Duration).

Finally, Economic Impact has been divided into Direct Effects (Direct Effects), more amenable to quantification, and Intangible Effects. The direct effects include the costs generated by the different bans or restrictions to normal living conditions, which can be sub-divided into Costs to the Economy, the direct economic impact of the restrictions, either in terms of the cost of the food affected by bans or in terms of loss of production (e.g., share in the fall of the gross domestic product), and Application Costs, i.e., direct cost of the application of chemical and physical remedial countermeasures: manpower, consumables, equipment needed for application, management of waste generated, etc.

As regards Intangible Effects, we consider the Cost of Image, which represents the indirect costs associated with the different strategies that can be perceived by the public differently due to market reluctance concerning even uncontaminated products, a drop in tourism, etc.

Next, attributes were established for the lowest-level objectives to indicate to what extent they were achieved by the respective remedial countermeasures. Table 1 shows the attribute names, units and ranges for seven out of the nine attributes under consideration using a continuous scale. Ordinal information about the component utilities of the countermeasures were considered for the remaining attributes, $X_{2}$ : Dose to Fish and $X_{9}$ : Cost to Image, as described afterwards.

\section{Group Decision-Making with Partial Information}

Eight different chemical, social and physical countermeasures were considered for analysis in the restoration problem under consideration:

- $A_{1}, A_{2}$ : Potassium addition These countermeasures aim at reducing $\mathrm{Cs}^{137}$ intake by fish. It is based upon the fact that $\mathrm{Cs}$ and $\mathrm{K}$ intakes are chemically competitive, and thus a greater amount of $\mathrm{K}$ tends to reduce $\mathrm{Cs}^{137}$ intake. $A_{1}$ consists of a 
Table 2 Countermeasure impacts

\begin{tabular}{llllllllll}
\hline & $A_{1}$ & $A_{2}$ & $A_{3}$ & $A_{4}$ & $A_{5}$ & $A_{6}$ & $A_{7}$ & $A_{8}$ & $A_{9}$ \\
\hline$X_{1}$ : L. Ecosyst. Index & 1.33 & 1.33 & 1.332 & 1.630 & 1.618 & 1.962 & 1.33 & 1.33 & 1.33 \\
$X_{3}$ : Dose Crit. Indiv. & 43.7 & 41.9 & 45.2 & 44.8 & 41.9 & 44.8 & 20.7 & 1.7 & 45.3 \\
$X_{4}$ : Collective Dose & 3490 & 3360 & 3630 & 3590 & 3360 & 3560 & 1690 & 180 & 3630 \\
$X_{5}$ : Amount of Fish & 0 & 0 & 0 & 0 & 0 & 0 & 0 & 36 & 0 \\
$X_{6}$ : Ban Duration & 0 & 0 & 0 & 0 & 0 & 0 & 0 & 120 & 0 \\
$X_{7}$ : Costs to Economy & 0 & 0 & 0 & 0 & 0 & 0 & 0 & 62,300 & 0 \\
$X_{8}:$ Application Cost & 2242 & 7830 & 1052 & 4209 & 11,807 & 38 & $31,56,390$ & 0 & 0 \\
\hline
\end{tabular}

single potassium treatment, whereas $A_{2}$ refers to a periodic potassium treatment (7 years).

- $A_{3}$ : Lake liming This countermeasure aims at reducing biological intake of $\mathrm{Cs}^{137}$ and $\mathrm{Sr}^{90}$. It is based upon the fact that $\mathrm{pH}$ influences the intake of those elements, and usually the higher the $\mathrm{pH}$, the lower the intake.

- $A_{4}, A_{5}$ : Wetland liming This countermeasure is similar to the above but the lime is added to the whole catchment. A single $\left(A_{4}\right)$ and a periodic $\left(A_{5}\right)$ catchment liming are considered.

- $A_{6}$ : Fertilization This countermeasure tries to cause a biological dilution of contamination, based upon the idea that, by increasing the biomass of the lake, the concentration of contamination in it must decrease.

- $A_{7}$ : Removal of contaminated bottom sediments A total clean-up of the sediment was assumed to take place 30 months after the contamination, affecting the top 10 $\mathrm{cm}$ layer.

- As: Treatment of contaminated fish and Bans on fish consumption There are several kinds of treatments, such as removing the bones or salting the fish. The first is quite effective for reducing $\mathrm{Sr}^{90}$ intake, whereas fish salting can reduce $\mathrm{Cs}^{137}$ by $70 \%$.

The above countermeasures were also compared with $A_{9}$ : No action, i.e., the natural evolution of the situation without intervention. Further information about the countermeasures under consideration can be found in Gallego and Magán (2009).

Table 2 shows the impacts or performances for the different attributes with a continuous scale for the nine countermeasures under consideration. These impacts were gathered from MOIRA system, which includes a complete set of reliable, validated models (Monte et al. 2009) to predict the long-term temporal behavior of radionuclides in the freshwater environment and the ecological, social and economic impacts of the intervention countermeasures.

Note that although the table includes precise impacts, percentage deviations were used to account for uncertainty about some such impacts. Specifically, a $20 \%$ deviation was introduced in $X_{1}$ and $X_{8}$, a $10 \%$ for $X_{5}$ and $X_{7}$; and deviations ranging from -10 to $+30 \%$ were used to derive the least and most impact for attributes $X_{3}$ and $X_{4}$, respectively.

Besides, DM preferences were modeled by an additive multi-attribute utility function, whose functional form is 


$$
u\left(A_{i}\right)=\sum_{j=1}^{n} w_{j} u_{j}\left(x_{i j}\right),
$$

where $x_{i j}$ is the impact of countermeasure $A_{i}$ with respect to attribute $X_{j}, u_{j}($.$) is the$ component utility function representing DM preferences for the impacts of attribute $X_{j}$ and $w_{j}$ is the weight representing the relative importance of each attribute. Note that $\sum_{j} w_{j}=1$ and $w_{j} \geq 0$.

Imprecision was considered regarding the DMs' preferences when assessing both the DMs' component utilities, $u_{j}($.$) , and weights. In the first case, classes of component$ utility functions were derived from the elicitation methods (Jiménez et al. 2003) and, consequently, the component utility associated with a specific impact would belong to an interval. Alternatively, ordinal information about the component utilities of the countermeasures was considered (Sarabando and Dias 2010; Aguayo et al. 2014), i.e., the DM provided a ranking of the countermeasures for some attributes. Moreover, rankings of the difference between the values of consecutive countermeasures was also taken into account for some attributes.

Besides, imprecision on weights representing the relative importance of criteria was represented by means of ordinal information.

Three experts participated in the ecosystem restoration problem solving: D. Cancio, former Head of the Public and Environmental Radiological Protection Unit (CIEMAT, Centro de Investigaciones Energéticas, Medioambientales y Tecnológicas-Energy, Environment and Technology Research Centre); P. Carboneras, former Director of the Safety and Licensing Department at ENRESA (Empresa Nacional de Residuos Radiactivos S.A.—National Radioactive Waste Management Company), and E. Gallego, Professor of Nuclear Engineering at the UPM and public and environmental radiological protection expert. Another expert assuming a more ecological role $\left(\mathrm{DM}_{4}\right)$ was also involved in the analysis.

The relative importance of the experts was as follows: the ecology expert was assigned a weight of 0.1 , whereas the other three experts were considered equally important as each other and each was assigned a weight of 0.3 .

Figure 2 shows the utility functions for $X_{1}$ : Lake Ecosystem Index and $X_{3}$ : Dose to Critical Individuals. An imprecise piecewise utility function was considered for $X_{3}$, whereas a precise nonlinear decreasing utility function was used for $X_{1}$ (Håkanson et al. 2000). For the remaining attributes, a precise linear decreasing utility function was considered in the corresponding attribute range.
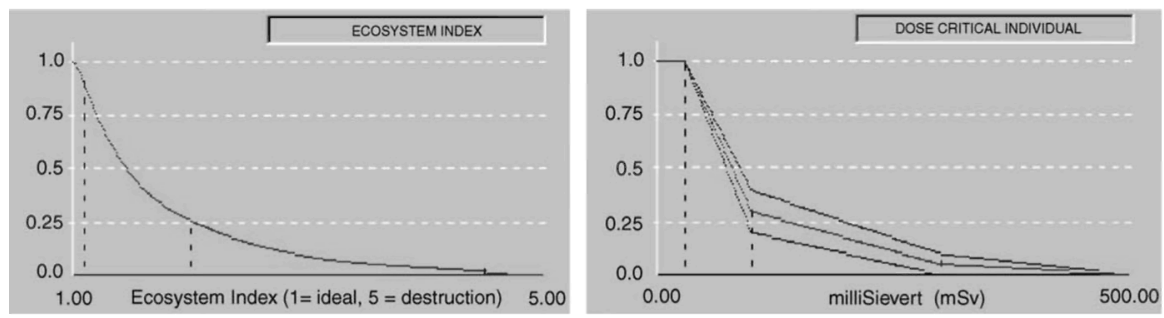

Fig. 2 Component utility functions for $X_{1}$ and $X_{3}$ 
Table 3 Ordinal information concerning countermeasures

Table 4 Ordinal information about the difference between the countermeasures

\begin{tabular}{ll}
\hline \multicolumn{2}{c}{ Ordinal information } \\
\hline$X_{2}:$ Dose to Fish & $A_{7}>\left\{A_{2}, A_{3}, A_{4}, A_{5}, A_{6}, A_{8}, A_{9}\right\}>A_{1}$ \\
$X_{9}:$ Cost to Image & \\
$D M_{1}$ & $\left\{A_{1}, A_{2}, A_{3}, A_{4}, A_{5}, A_{6}\right\}>A_{8}>A_{7}$ \\
$D M_{2}$ & $A_{7}>\left\{A_{1}, A_{2}, A_{3}, A_{4}, A_{5}, A_{6}\right\}>A_{8}>A_{9}$ \\
$D M_{3}$ & $A_{7}>\left\{A_{2}, A_{5}\right\}>\left\{A_{1}, A_{3}, A_{4}, A_{6}\right\}>A_{8}>A_{9}$ \\
$D M_{4}$ & $\left\{A_{6}, A_{7}, A_{8}\right\}>\left\{A_{3}, A_{4}\right\}>\left\{A_{1}, A_{2}, A_{5}\right\}>A_{9}$ \\
\hline
\end{tabular}

\begin{tabular}{ll}
\hline & Ordinal information \\
\hline$X_{2}:$ Dose to Fish & $\Delta_{2\{7,2\}}>\Delta_{2\{9,1\}}$ \\
$X_{9}:$ Cost to Image & \\
$D M_{1}$ & $\Delta_{9\{8,7\}}>\Delta_{9\{6,8\}}$ \\
$D M_{2}$ & $\Delta_{9\{7,1\}>\Delta_{9\{8,9\}}>\Delta_{9\{6,8\}}}$ \\
$D M_{3}$ & $\Delta_{9\{7,2\}}>\Delta_{9\{8,9\}}>\left\{\Delta_{9\{5,1\}}, \Delta_{9\{6,8\}}\right\}$ \\
\hline
\end{tabular}

Table 3 shows the ordinal information concerning the appraisal of the nine countermeasures in $X_{2}$ : Dose to Fish and $X_{9}$ : Cost to Image. Note that as $X_{9}$ : Cost to Image is subjective, ordinal information for each DM was incorporated to the analysis, whereas the ordinal information concerning the appraisal of $X_{2}$ : Dose to Fish was not subjective and ordinal information accounting for all the DMs was considered. Note also that countermeasures in braces were equally valued.

Moreover, rankings of the difference between the values of some consecutive countermeasures were also taken into account by DMs for some attributes, see Table 4.

For $X_{2}$ : Dose to Fish, we have $A_{7}>\left\{A_{2}, A_{3}, A_{4}, A_{5}, A_{6}, A_{8}, A_{9}\right\}>A_{1}$ and $\Delta_{2\{7,2\}}>\Delta_{2\{9,1\}}$, where

$$
\begin{array}{ll}
\Delta_{2\{7,2\}}=v_{2}\left(A_{7}\right)-v_{2}\left(A_{2}\right), & \Delta_{2\{2,3\}}=v_{2}\left(A_{2}\right)-v_{2}\left(A_{3}\right)=0, \\
\Delta_{2\{3,4\}}=v_{2}\left(A_{3}\right)-v_{2}\left(A_{4}\right)=0, & \Delta_{2\{4,5\}}=v_{2}\left(A_{4}\right)-v_{2}\left(A_{5}\right)=0, \\
\Delta_{2\{5,6\}}=v_{2}\left(A_{5}\right)-v_{2}\left(A_{6}\right)=0, & \Delta_{2\{6,8\}}=v_{2}\left(A_{6}\right)-v_{2}\left(A_{8}\right)=0, \\
\Delta_{2\{8,9\}}=v_{2}\left(A_{8}\right)-v_{2}\left(A_{9}\right)=0, & \Delta_{2\{9,1\}}=v_{2}\left(A_{9}\right)-v_{2}\left(A_{1}\right) .
\end{array}
$$

This means that the difference in the appraisal of countermeasure $A_{7}$ regarding $\left\{A_{2}, A_{3}, A_{4}, A_{5}, A_{6}, A_{8}, A_{9}\right\}$ is greater than the difference in the appraisal of that set regarding $A_{1}$.

For $X_{9}$ : Cost to Image, we have for $D M_{3} A_{7}>\left\{A_{2}, A_{5}\right\}>\left\{A_{1}, A_{3}, A_{4}, A_{6}\right\}>$ $A_{8}>A_{9}$ and $\Delta_{9\{7,2\}}>\Delta_{9\{8,9\}}>\left\{\Delta_{9\{5,1\}}, \Delta_{9\{6,8\}}\right\}$, where

$$
\begin{array}{ll}
\Delta_{9\{7,2\}}=v_{9}\left(A_{7}\right)-v_{9}\left(A_{2}\right), & \Delta_{9\{2,5\}}=v_{9}\left(A_{2}\right)-v_{9}\left(A_{5}\right)=0, \\
\Delta_{9\{5,1\}}=v_{9}\left(A_{5}\right)-v_{9}\left(A_{1}\right), & \Delta_{9\{1,3\}}=v_{9}\left(A_{1}\right)-v_{9}\left(A_{3}\right)=0,
\end{array}
$$


Table 5 Ordinal information concerning weights

\begin{tabular}{ll}
\hline & Ordinal information \\
\hline$D M_{1}$ & $w_{3}^{1}>w_{8}^{1}>w_{9}^{1}>w_{4}^{1}>\left\{w_{5}^{1}, w_{6}^{1}\right\}>w_{7}^{1}>w_{2}^{1}>w_{1}^{1}$ \\
$D M_{2}$ & $w_{3}^{2}>w_{4}^{2}>w_{9}^{2}>w_{6}^{2}>w_{8}^{2}>w_{7}^{2}>w_{1}^{2}>w_{2}^{2}>w_{5}^{2}$ \\
$D M_{3}$ & $\left\{w_{3}^{3}, w_{4}^{3}\right\}>w_{8}^{3}>w_{6}^{3}>w_{9}^{3}>w_{7}^{3}>\left\{w_{1}^{3}, w_{2}^{3}, w_{5}^{3}\right\}$ \\
$D M_{4}$ & $\left\{w_{1}^{4}, w_{2}^{4}\right\}>\left\{w_{3}^{4}, w_{4}^{4}\right\}>\left\{w_{5}^{4}, w_{6}^{4}\right\}>\left\{w_{7}^{4}, w_{8}^{4}\right\}>w_{9}^{4}$ \\
\hline
\end{tabular}

Table 6 Ordinal information about the difference between the weights

\begin{tabular}{ll}
\hline DM & Ordinal information \\
\hline$D M_{1}$ & $\Delta_{1\{3,8\}}>\Delta_{1\{9,4\}}>\left\{\Delta_{1\{6,7\}}, \Delta_{1\{7,2\}}\right\}>\left\{\Delta_{1\{8,9\}}, \Delta_{1\{4,5\}}, \Delta_{1\{2,1\}}\right\}$ \\
$D M_{2}$ & $\Delta_{2\{1,2\}}>\Delta_{2\{2,5\}}>\Delta_{2\{4,9\}}>\left\{\Delta_{2\{3,4\}}, \Delta_{2\{9,6\}}, \Delta_{2\{6,8\}}, \Delta_{2\{8,7\}}, \Delta_{2\{7,1\}}\right\}$ \\
$D M_{3}$ & $\Delta_{3\{4,8\}}>\Delta_{3\{8,6\}}>\Delta_{3\{6,9\}}>\Delta_{3\{9,7\}}>\Delta_{3\{7,1\}}$ \\
\hline
\end{tabular}

$$
\begin{array}{ll}
\Delta_{9\{3,4\}}=v_{9}\left(A_{3}\right)-v_{9}\left(A_{4}\right)=0, & \Delta_{9\{4,6\}}=v_{9}\left(A_{4}\right)-v_{9}\left(A_{6}\right)=0, \\
\Delta_{9\{6,8\}}=v_{9}\left(A_{6}\right)-v_{9}\left(A_{8}\right), & \Delta_{9\{8,9\}}=v_{9}\left(A_{8}\right)-v_{9}\left(A_{9}\right) .
\end{array}
$$

In this case, the difference in the appraisal of countermeasure $A_{7}$ regarding $\left\{A_{2}, A_{5}\right\}$ is greater than the difference in the appraisal of countermeasures $A_{9}$ and $A_{8}$, which is greater than the other two differences in appraisal.

Each DM also used ordinal information to represent imprecision with respect to weights in the restoration problem under consideration, as shown in Table 5.

Rankings of the difference between the values of some consecutive weights were also taken into account by some DMs, see Table 6 .

\section{Veto in MCDM}

The concept of veto was originally justified in social theory by the prudence axiom enunciated by Arrow and Raynaud (1986). The main idea behind this axiom is that it is not prudent to accept highly conflicting alternatives that may result in vulnerable decisions. Regarding the prudence axiom, Moulin defines the principle of proportional veto within a group of DMs (Moulin 1981), according to which any coalition of DMs should be given the right to veto a certain number of alternatives, which is approximately proportional to the size of this coalition. In addition, the allocation of veto power across the various groups of social participants has ethical implications, since it entails attaching different weights to different groups.

In MCDM problems the concept of veto has been used to manage non-compensatory methods. In outranking methods the use of veto usually represents the intensity of preference of the minority (Roy and Slowinski 2008). For instance, Nowak (2004) used ELECTRE-III to build a multi-attribute ranking using preference thresholds to distinguish situations of strict and weak preference in stochastic dominance approaches. 
Later, Munda (2009) implemented a veto-based threshold to deal with environmental and resource management and policies aimed at sustainable development. A fuzzy set theory framework was used to represent qualitative information by means of the concept of linguistic variable. Ranking policy options were derived by means of the majority principle implemented by Concordet, whereas the power of a subgroup of DMs to veto some alternatives was accounted for by means of Moulin's proportional veto function.

More recently, Greco et al. (2011) proposed a new method, called ELECTRE GKMS. ELECTRE ${ }^{G K M S}$ employs robust ordinal regression to construct a set of outranking models compatible with preference information. It builds a set of values of concordance indices, concordance thresholds, indifference, preference, and veto thresholds, for which all specified pairwise comparisons can be restored.

On the other hand, additive compensatory methods have also incorporated the concept of veto. For instance, Bana e Costa et al. (2002) define a multi-criteria approach for prohibiting alternatives by the measuring attractiveness by a categorical based evaluation technique (MACBETH) for facilitating bid evaluation processes, such as interventions in an international public call for tenders. The result is a procedure called the determinants technique, whose groundwork is aligned, albeit not directly, with the notion of veto power used to model non-compensatory situations.

In connection with research based on the power of veto, Marichal (2004) proposes axiomatizing individual indices to rate whether each criterion behaves as a veto or an aggregator using the Choquet integral. These indices for measuring the degree to which each criterion behaves like a blocker or a pusher make it possible to identify and measure the dictatorial tendency of criteria, which is a particular interaction phenomenon. Here, the veto is not a preference parameter given by the DM but an effect phenomenon when aggregating criteria. Therefore, the veto concept is related to the impact caused by a criterion on the global evaluation of alternatives.

Liginlala and Ow (2006) use the same idea of veto effects, expressing degrees of conjunction, disjunction, veto and approval given by the indices through fuzzy analysis measures, which represent a risk tolerance measure of the DM. The veto power examines how tolerant DMs are about accepting or rejecting evaluations of alternatives associated with specific actions on a given attribute.

Daher and de Almeida (2012) developed an additive group preference model that incorporates a utility reduction factor. DMs express their preferences in terms of a ranking of alternatives and are able to make an informed veto by providing information about the undesirable or unacceptable ranking of some alternatives. The ranking veto is achieved by using a reduction factor on the global utility of the alternatives.

More recently, de Almeida (2013) proposed two additive-veto models for choice and ranking problems, respectively. The level of veto is represented by two thresholds. This introduces some vagueness in the DM's specification of this level.

Then, if a choice problem is considered, veto functions of all attributes are aggregated by the product function and incorporated into the additive model and the global value/utility is set to 0 if the performance of the alternative is unacceptable for any of the attributes. In ranking problems, the veto function rejects the position of the vetoed alternative in the ranking process rather than the alternative itself. Weight veto functions are aggregated by summation and incorporated into the additive model. 
In this paper, as in de Almeida (2013), we propose an additive-veto model for ranking problems, albeit in a group decision-making context. Veto values provided by DMs are precise and expressed in terms of alternative performances. Then, veto values for the most important DMs are used to define veto ranges, whereas veto values corresponding to the other less important DMs are partially taken into account, leading to the construction of adjust ranges.

Approaches by Daher and de Almeida (2012) and de Almeida (2013) are described in more detail and compared with the use of veto values in the group decision-making context with partial information as proposed in this paper in Sect. 6.

\subsection{Accounting for Veto Values in a Group Decision-Making Context}

We now extend the decision-making framework with the incomplete information to a group decision-making context in which DMs are allowed to provide vetoes for the alternative performances with respect to the attributes under consideration.

Then, we consider a set of $k$ DMs, denoted by $D M_{l}, l=1, \ldots, k$, whose relative importance is known and denoted by $w_{D M_{l}}$. Without loss of generality, we assume that the most important DM is $D M_{1}$, followed by $D M_{2}$, and so on up to $D M_{k}$. Consequently, $w_{D M_{1}} \geq w_{D M_{2}} \geq \cdots \geq w_{D M_{k}}$, and $\sum_{l} w_{D M_{l}}=1$.

The question of how to measure the weights of DMs in a group decision-making context is an interesting research topic. Yue (2011) provides a brief overview of approaches proposed by different authors to determine the weights of DMs. Besides, a new approach based on an extended TOPSIS method (Yoon 1980; Hwang and Yoon 1981 ) is also proposed.

We assume that weight intervals or ordinal information about weights are available for each $\mathrm{DM}, \mathbf{w}^{l}=\left(w_{1}^{l}, \ldots, w_{n}^{l}\right) \in W^{l}, l=1, \ldots, k$, representing the relative importance of their attributes. Moreover, imprecise component utility functions have been built or ordinal information about the utility of the alternatives has been provided by the DMs for the attributes under consideration, $U_{j}^{l}, l=1, \ldots, k$ and $j=1, \ldots, n$.

All DMs are allowed to provide veto values, but the corresponding veto will be effective for only the $r$ most important DMs, $r \leq k$. Veto values corresponding to the $k-r$ remaining DMs will be partially taken into account, as described later.

We denote by $v_{j}^{l}$ the veto threshold provided by the $l$-th DM for the attribute $X_{j}$, i.e. the $l$-th DM wants the alternative performances to be greater (less) than $v_{j}^{l}$ if an increasing (decreasing) component utility function is associated with attribute $X_{j}$. Consequently, the veto interval for the $l$-th DM is $\left(0, v_{j}^{l}\right]$ in attribute $X_{j}$. For simplicity's sake, we will consider from now on that component utility functions are increasing.

A veto range can then be identified for each attribute $\left[v_{j}^{L}, v_{j}^{U}\right]$, where $v_{j}^{L}=r_{j}^{L}$, $\left[r_{j}^{L}, r_{j}^{U}\right]$ being the attribute range, and $v_{j}^{U}=\max _{l=1, \ldots, r}\left\{v_{j}^{l}\right\}$, i.e., the highest veto value for attribute $X_{j}$ for the $r$ most important DMs.

We build an adjust range for each attribute $X_{j},\left(a_{j}^{L}, a_{j}^{U}\right.$, with $a_{j}^{L}=v_{j}^{U}$, and $a_{j}^{U}=\max _{l=1, \ldots, k}\left\{v_{j}^{l}\right\}$, i.e., the highest veto value for attribute $X_{j}$ considering all DMs. 
Fig. 3 Adjust function

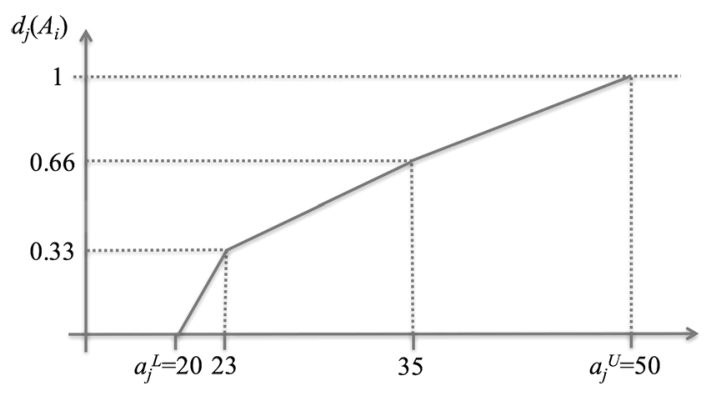

We add the above information to the additive multi-attribute utility function by means of the following functions (assuming increasing component utility functions):

- $v\left(A_{i}\right)$ is the veto function that checks if the performances for a given $A_{i}$ are within the respective veto intervals:

$$
v\left(A_{i}\right)=\prod_{j=1}^{n} v_{j}\left(A_{i}\right), \quad \text { with } v_{j}\left(A_{i}\right)=\left\{\begin{array}{l}
1, \text { if } x_{i j}>v_{j}^{U} \\
0, \text { if } x_{i j} \leq v_{j}^{U}
\end{array}\right.
$$

Note that $v\left(A_{i}\right)=0$ if at least one performance is within the veto interval for the corresponding attribute.

- $d_{j}\left(A_{i}\right)$ is the adjust function that decreases the utility associated with the alternative performances within the corresponding adjust range. A first possible approach is to apply a linear adjust function. However, we believe that the veto values for the $k-r$ less important DMs should be added by means of this adjust function. Veto values provided by the $k-r$ DMs may be within the adjust interval. In this case, we use this information to build a piecewise linear function.

For example, let us assume that the adjust range is [20, 50], 50 being the highest veto value provided by the DMs. Then, if two of the $k-r$ less important DMs have provided the veto values 23 and 35, i.e. veto values within the adjust range, then the corresponding adjust function is as shown in Fig. 3. Note that the adjust values for the considered vetoes are 0.33 and 0.66 , respectively. In case of three veto values within the adjust range, their adjust values would be $0.25,0.5$ and 0.75 .

Table 7 shows the veto values provided by the DMs for the restoration problem under consideration. Note that, DMs do not provide veto values for all attributes, and the only veto for attribute $X_{6}$ is provided by $D M_{1}$. Besides, as component utility functions are decreasing for all three attributes, the vetoed values are the values greater than the veto values provided by DMs. Note that the respective veto will be effective for the three most important DMs only, i.e., $D M_{1}, D M_{2}$ and $D M_{3}$.

The veto intervals for $X_{1}, X_{3}$ and $X_{6}$ are [1.7, 5], [100, 500] and [24, 360], respectively; whereas adjust intervals are $[1.6,1.7]$ and $[90,100]$, corresponding to attributes $X_{1}$ and $X_{3}$, i.e., no adjust interval is identified for $X_{6}$. Thus, countermeasure $A_{6}:$ Fertilization is vetoed since its impact interval for $X_{1}$ is $[1.766,2.354]$ within the veto 
Table 7 Veto values for DMs

\begin{tabular}{llll}
\hline $\mathrm{DM}$ & $X_{1}(\mathrm{LEI})$ & $X_{3}(\mathrm{mSv})$ & $X_{6}$ (months) \\
\hline$D M_{1}$ & - & 100 & 24 \\
$D M_{2}$ & 1.7 & 100 & - \\
$D M_{3}$ & 2 & - & - \\
$D M_{4}$ & 1.6 & 90 & - \\
\hline
\end{tabular}
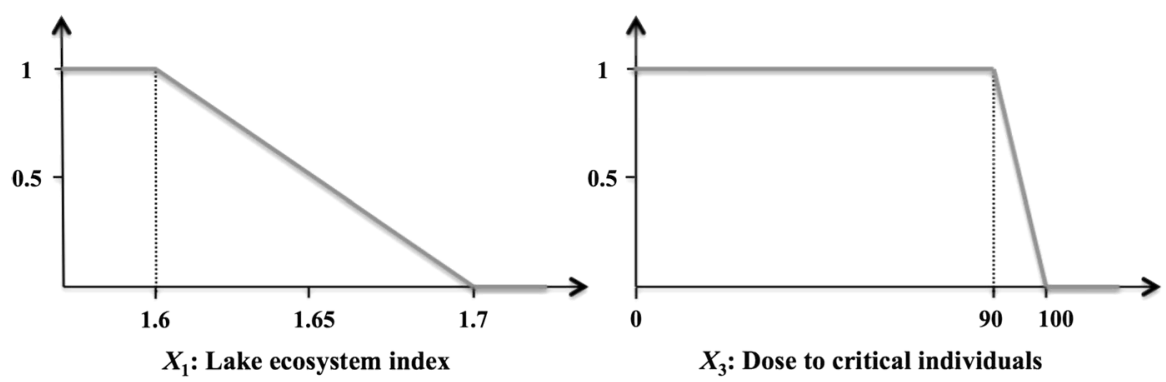

Fig. 4 Adjust functions for $X_{1}$ and $X_{3}$

interval $[1.7,5]$. A8: Treatment of contaminated fish and Bans on fish consumption is also vetoed since its impact on $X_{6}$ is 36 , which falls within the veto interval $[24,360]$. Consequently, neither countermeasure will be considered for further analysis.

The adjust functions for attributes $X_{1}$ and $X_{3}$ are shown in Fig. 4. The upper endpoint of the performance interval for countermeasure $A_{1}$ : Single Potassium addition with respect to $X_{1}, 1.609$, is within the corresponding adjust interval. Thus, the original component utility, 0.4 , is reduced according to the adjust function leading to $0.4 \times$ $0.91=0.364$. The same applies to $A_{3}$ : Lake liming, whose reduced lower component utility in $A_{1}$ is $0.41 \times 0.88=0.3608$. Note that the adjust functions have little effect on the evaluation of alternatives in the problem under consideration, but this might not be the case in other decision-making contexts.

\section{Group Decision-Making Within MAUT with Incomplete Information Accounting for Veto}

The adaptation of the additive multi-attribute utility function to account for the veto and adjust functions is as follows:

$$
u^{l}\left(A_{i}\right)=\left[\sum_{j=1}^{n} u_{j}^{l}\left(x_{i j}\right) w_{j}^{l} d_{j}\left(A_{i}\right)\right] \times v\left(A_{i}\right),
$$

with

$$
\mathbf{w}^{l} \in W^{l}=\left\{\left(w_{1}^{l}, \ldots, w_{n}^{l}\right) \mid w_{(1)}^{l} \geq w_{(2)}^{l} \geq \cdots \geq w_{(n)}^{l} \geq 0\right\}
$$


i.e. ordinal information regarding the relative importance of criteria $\left(\sum_{j=1}^{n} w_{i}=1\right)$ and $d_{j}\left(A_{i}\right)$ and $v\left(A_{i}\right)$ are the adjust and veto functions, respectively, as described in Sect. 4.1.

Regarding component utilities, classes of component utility functions could be derived from the elicitation methods (Jiménez et al. 2003). This applies to the attributes in Table 2. Then,

$\mathbf{u}_{j} \in U_{j}=\left\{\left(u_{j}\left(x_{1 j}\right), \ldots, u_{j}\left(x_{m j}\right)\right) \mid u_{j}^{L}\left(x_{i j}^{L}\right) \leq u_{j}\left(x_{i j}\right) \leq u_{j}^{U}\left(x_{i j}^{U}\right), \quad i=1, \ldots, m\right\}$,

or

$\mathbf{u}_{j} \in U_{j}=\left\{\left(u_{j}\left(x_{1 j}\right), \ldots, u_{j}\left(x_{m j}\right)\right) \mid u_{j}^{L}\left(x_{i j}^{U}\right) \leq u_{j}\left(x_{i j}\right) \leq u_{j}^{U}\left(x_{i j}^{L}\right), \quad i=1, \ldots, m\right\}$,

for an increasing or decreasing utility function, respectively.

Alternatively, ordinal information about the component utilities of the alternatives could be provided by the DMs (Sarabando and Dias 2010; Aguayo et al. 2014). This applies to $X_{2}$ : Dose to Fish and $X_{9}$ : Cost to Image, see Table 3 . Then,

$\mathbf{u}_{j}^{l} \in U_{j}^{l}=\left\{\left(u_{j}\left(x_{1 j}\right)^{l}, \ldots, u_{j}\left(x_{m j}\right)^{l}\right) \mid u_{j}\left(x_{(1) j}\right)^{l} \geq u_{j}\left(x_{(2) j}\right)^{l} \geq \ldots \geq u_{j}\left(x_{(m) j}\right)^{l}\right\}$.

Moreover, rankings of the difference between the values of consecutive alternatives has been provided for both attributes, see Table 4 .

Note that although the above expression considers the preferences concerning weights and some component utilities regarding the $l$-th DM, the resulting utility is influenced by the opinion of the other DMs by means of the veto and adjust functions.

We now propose a methodology accounting for this specific group decision-making problem to derive a group ranking. The methodology consists of two aggregation phases. In the first phase, a dominance measuring method is used to exploit the uncertainty about the countermeasure impacts and imprecise information concerning DM preferences to derive a ranking of alternatives for each DM. In the second phase, the different rankings derived in the first phase are aggregated taking into account the relative importance of DMs to reach a group ranking using the order explicit algorithm method, an extension of Kemeny optimal aggregation.

\subsection{A Dominance Measuring Method for Deriving Alternative Rankings}

Dominance measuring methods (DMMS) are based on the computation of a dominance matrix, $D$, including pairwise dominance values. Dominance matrix for the $l$-th DM, $D^{l}$, can be defined as follows: 


$$
D^{l}=\left(\begin{array}{ccccc}
- & D_{12}^{l} & \cdots & D_{1(m-1)}^{l} & D_{1 m}^{l} \\
D_{21}^{l} & - & \cdots & D_{2(m-1)}^{l} & D_{2 m}^{l} \\
D_{31}^{l} & D_{32}^{l} & \cdots & D_{3(m-1)}^{l} & D_{3 m}^{l} \\
\vdots & \vdots & \vdots & \vdots & \vdots \\
D_{m 1}^{l} & D_{m 2}^{l} & \cdots & D_{m(m-1)}^{l} & -
\end{array}\right)
$$

where

$$
\begin{array}{ll}
D_{k s}^{l}= & \min \left\{u^{l}\left(A_{k}\right)-u^{l}\left(A_{s}\right)\right\} \\
\text { s.t. } & \\
& u_{j}\left(x_{k j}\right), u_{j}\left(x_{s j}\right) \in U_{j}, \quad j=1,3,4,5,6,7,8 \\
& u_{j}^{l}\left(x_{k j}\right), u_{j}^{l}\left(x_{s j}\right) \in U_{j}^{l}, \quad j=2,9, \\
& \mathbf{w}^{l}=\left(w_{1}^{l}, \ldots, w_{n}^{l}\right) \in W^{l},
\end{array}
$$

with

$$
u^{l}\left(A_{i}\right)=\left[\sum_{j=1}^{n} u_{j}^{l}\left(x_{i j}\right) w_{j}^{l} d_{j}\left(A_{i}\right)\right] \times v\left(A_{i}\right) .
$$

Note that given two alternatives $A_{k}$ and $A_{s}$, alternative $A_{k}$ dominates $A_{s}$ if $D_{k s}^{l} \geq 0$, and there exists at least one combination of values for the imprecise weights and component utilities under consideration such that the overall value of $A_{k}$ is strictly greater than that of $A_{s}$. This concept of dominance is called pairwise dominance. We have to solve quadratic optimization problems in order to compute pairwise dominance values.

Different DMMs can be found in the literature (Ahn and Park 2008; Jiménez et al. 2013; Aguayo et al. 2014; Mateos et al. 2014; Aguayo 2014). They differ as to how they exploit the information included in the dominance matrix to derive the ranking of alternatives. Their performance has been compared with other approaches to deal with incomplete information, such as the modification of four classical decision rules to encompass an imprecise decision context (Puerto et al. 2000; Salo and Hämäläinen 2001), surrogate weighting methods (Barron and Barret 1996; Stillwell et al. 1981), the stochastic multicriteria acceptability analysis (SMAA) method (Lahdelma et al. 1998) and its extensions, SMAA-2 (Lahdelma and Salminen 2001) and SMAA-O (Lahdelma et al. 2003); or Sarabando and Dias' method (2009).

Most recent $D M M S$ outperfom most of the approaches under comparison. Besides, approaches for which there was no significant difference regarding $D M M S, R O C$ (a surrogate weighting method), SMAA-2 and Sarabando and Dias' method, have the drawback of only being applicable for specific ways of representing the imprecise information about the DMs' preferences, whereas DMMS can incorporate and combine different ways of representing such imprecision, as illustrated in the example introduced in Sect. 4. 
The DMM that we use Aguayo (2014) derives a global dominance intensity index to rank alternatives on the basis that

$$
D_{k s}^{l} \leq u^{l}\left(A_{k}\right)-u^{l}\left(A_{s}\right) \leq\left|D_{s k}^{l}\right|
$$

First, the pairwise dominance values in $D^{l}$ are weighted according to the distance between the central weight vector $\left(w_{j}^{l c}\right)$ and the weight vector $\left(w_{j}^{l *}\right)$ associated with the optimal $D_{k s}^{l}$, when solving the corresponding optimization problem, i.e.,

$$
\bar{D}_{k s}^{l}=\frac{D_{k s}^{l}}{d\left(w_{j}^{l c}, w_{j}^{l *}\right)} .
$$

The aim is to attach more importance to weight vectors closer to the central weight vector.

Then, we perform the following algorithm:

1. If $\bar{D}_{k s}^{l} \geq 0$, then alternative $A_{k}$ dominates $A_{s}$, and the dominance intensity of $A_{k}$ over $A_{s}$ is $D I_{k s}^{l}=d\left(\left[\bar{D}_{k s}^{l},-\bar{D}_{s k}^{l}\right], 0\right)$.

Else $\left(\bar{D}_{k s}^{l}<0\right)$ :

- If $\bar{D}_{s k}^{l} \geq 0$, then $A_{s}$ dominates $A_{k}$, and $D I_{k s}^{l}=-d\left(\left[\bar{D}_{k s}^{l},-\bar{D}_{s k}^{l}\right], 0\right)$.

- Else $\left(\bar{D}_{s k}^{l}<0\right)$,

$$
D I_{k s}^{l}=\left[\frac{-\bar{D}_{s k}^{l}}{-\bar{D}_{s k}^{l}-\bar{D}_{k s}^{l}}-\frac{-\bar{D}_{k s}^{l}}{-\bar{D}_{s k}^{l}-\bar{D}_{k s}^{l}}\right] \times d\left(\left[\bar{D}_{k s}^{l},-\bar{D}_{s k}^{l}\right], 0\right) .
$$

2. Calculate a global dominance intensity $\left(G D I^{l}\right)$ for each alternative $A_{k}$, i.e., $G D I_{k}^{l}=\sum_{s=1, s \neq k}^{m} D I_{k s}^{l}$, and rank the alternatives according to them.

Note that the method incorporates the distance from the intervals $\left[\bar{D}_{k s}^{l},-\bar{D}_{s k}^{l}\right]$ to 0 to account for their sizes and how far they are from 0 . The distance $d$ used was proposed in Mateos et al. (2007), which takes into account every point in the interval.

For the restoration problem under consideration, the corresponding quadratic optimization problems were solved to compute pairwise dominance values and derive the following dominance matrices for the four DMs under consideration:

$$
D^{1}=\left(\begin{array}{ccccccc}
- & -0.1569 & -0.1746 & -0.1362 & -0.1042 & -0.1466 & -0.1272 \\
-0.1956 & - & -0.2363 & -0.2169 & -0.1866 & -0.2189 & -0.2607 \\
-0.1924 & -0.1806 & - & -0.2257 & -0.1179 & -0.0966 & -0.1823 \\
-0.2105 & -0.2034 & -0.1794 & - & -0.1949 & -0.1832 & -0.2814 \\
-0.2203 & -0.1662 & -0.1669 & -0.1895 & - & -0.1793 & -0.1953 \\
-0.2000 & -0.1484 & -0.1539 & -0.1570 & -0.0876 & - & -0.1591 \\
-0.1762 & -0.0972 & -0.1589 & -0.1914 & -0.1214 & -0.1139 & -
\end{array}\right),
$$


Table 8 Global dominance intensities and countermeasure rankings for DMs

\begin{tabular}{llllll}
\hline & $D M_{1}$ & $D M_{2}$ & $D M_{3}$ & $D M_{4}$ & Group \\
\hline $1 s t$ & $A_{9}(0.1162)$ & $A_{1}(0.2398)$ & $A_{1}(0.1904)$ & $A_{1}(0.2932)$ & $A_{1}$ \\
$2 n d$ & $A_{1}(0.1094)$ & $A_{7}(0.1506)$ & $A_{3}(0.1453)$ & $A_{3}(0.1427)$ & $A_{9}$ \\
$3 r d$ & $A_{3}(0.0235)$ & $A_{9}(0.0278)$ & $A_{9}(0.1419)$ & $A_{2}(0.0163)$ & $A_{3}$ \\
$4 t h$ & $A_{7}(0.0125)$ & $A_{3}(-0.0051)$ & $A_{7}(0.0305)$ & $A_{9}(-0.0494)$ & $A_{7}$ \\
$5 t h$ & $A_{4}(-0.0419)$ & $A_{4}(-0.1158)$ & $A_{4}(-0.0492)$ & $A_{7}(-0.0843)$ & $A_{4}$ \\
$6 t h$ & $A_{5}(-0.1006)$ & $A_{2}(-0.1362)$ & $A_{2}(-0.1811)$ & $A_{5}(-0.1022)$ & $A_{2}$ \\
$7 t h$ & $A_{2}(-0.1193)$ & $A_{5}(-0.1611)$ & $A_{5}(-0.2778)$ & $A_{4}(-0.2164)$ & $A_{5}$ \\
\hline
\end{tabular}

$$
\begin{aligned}
& D^{2}=\left(\begin{array}{ccccccc}
- & -0.1572 & -0.1553 & -0.2035 & -0.0298 & -0.1567 & -0.1713 \\
-0.3054 & - & -0.2034 & -0.2985 & -0.2120 & -0.3098 & -0.3407 \\
-0.2348 & -0.1991 & - & -0.1825 & -0.2397 & -0.3225 & -0.2338 \\
-0.2648 & -0.3035 & -0.2591 & - & -0.2763 & -0.3061 & -0.2371 \\
-0.3146 & -0.1970 & -0.3190 & -0.1922 & - & -0.2523 & -0.1846 \\
-0.1705 & -0.1095 & -0.2250 & -0.1849 & -0.1967 & - & -0.2134 \\
-0.2107 & -0.3127 & -0.2365 & -0.2039 & -0.1255 & -0.1981 & -
\end{array}\right), \\
& D^{3}=\left(\begin{array}{ccccccc}
- & -0.1174 & -0.1475 & -0.2228 & -0.06158 & -0.1918 & -0.1567 \\
-0.3291 & - & -0.2416 & -0.1875 & -0.2513 & -0.2255 & -0.3403 \\
-0.2314 & -0.0780 & - & -0.1416 & -0.1506 & -0.1905 & -0.1855 \\
-0.1979 & -0.2553 & -0.2857 & - & -0.1075 & -0.3162 & -0.2131 \\
-0.2425 & -0.2714 & -0.2851 & -0.3056 & - & -0.2456 & -0.2689 \\
-0.2255 & -0.1777 & -0.2300 & -0.1620 & -0.1776 & - & -0.2113 \\
-0.1836 & -0.1836 & -0.2005 & -0.1743 & -0.1071 & -0.1094 & -
\end{array}\right), \\
& D^{4}=\left(\begin{array}{ccccccc}
- & 0.0897 & -0.0707 & -0.0923 & 0.0078 & -0.0006 & -0.0099 \\
-0.1349 & - & -0.0302 & -0.0995 & -0.1138 & -0.0257 & -0.1489 \\
-0.1206 & -0.0193 & - & -0.0456 & 0.0249 & -0.0461 & -0.1712 \\
-0.0770 & -0.2456 & -0.1263 & - & -0.1095 & -0.2414 & -0.0706 \\
-0.0511 & -0.1594 & -0.1666 & -0.0631 & - & -0.0197 & -0.1137 \\
-0.0671 & -0.2201 & -0.1444 & 0.0183 & -0.0958 & - & -0.0525 \\
-0.1946 & -0.0665 & -0.1154 & -0.1351 & -0.1117 & -0.0043 & -
\end{array}\right) .
\end{aligned}
$$

Table 8 shows the global dominance intensities (GDIs) associated with each countermeasure and the resulting rankings for the DMs under consideration. Note that it would be wrong to refer to such rankings as individual rankings or as the ranking representing the preferences of the $l$-th DM, as the resulting utilities are influenced by the opinion of other DMs in the extension of the additive multi-attribute utility model to account for veto values.

\subsection{Aggregating Alternative Rankings}

In the second phase of the proposed methodology, the $k$ rankings output in the first phase have to be aggregated. As mentioned in the introduction, different meth- 
ods for aggregating rankings by different authors can be found in the literature (Lin 2010).

In our decision-making scenario, complete rankings and the relative importance of such rankings (relative importance of DMs) are available. Moreover, the values that lead to the corresponding rankings (global dominance intensities) derived from the dominance measuring method are also available. The only aggregation method to exploit all the above information is the Kemeny method (1959) and its extensions.

Kemeny optimal aggregation optimizes the average Kendall distances between a candidate aggregate ranking and each of the input rankings. As computing the Kemeny optimal aggregate is NP-hard even when the number of ranked lists to be aggregated is small, we have used the order explicit algorithm (OEA) Lin and Ding (2009) to solve the combinatorial problem under consideration.

OEA uses a global optimization technique, called the cross-entropy Monte Carlo method, which searches iteratively for an optimal list that minimizes a criterion, the sum of weighted distances between the candidate (aggregate) list and each of the input ranked lists. The method is, however, general and amenable to any other optimization criterion. A modified Kendall's tau measure and Spearman's footrule are used to measure the distance between two ranked lists.

The OEA algorithm has been implemented using R. The program and documentation are available at http://www.stat.osu.edu/?statgen/TopKCEMC. RankAggreg Pihur et al. (2009) is another R package for weighted rank aggregation including OEA, https://cran.r-project.org/web/packages/RankAggreg/RankAggreg.pdf.

The last column of Table 8 shows the resulting group ranking, in which the relative importance of DMs has been taken into account. Note that alternative $A_{1}$ : Potassium addition (single treatment) is best ranked in the group ranking, followed by $A_{9}:$ No action and $A_{3}$ : Lake liming. Alternative $A_{1}$ was best ranked by all DMs but $D M_{1}$, who placed it second.

\section{Comparative Analysis}

As cited in Sect. 4, de Almeida (2013) proposed two additive-veto models for choice and ranking problems, respectively, in which some vagueness was introduced in the DM's specification of veto values by two thresholds. In fact, the DM is willing to accept an alternative whose value/utility is above the upper threshold, the DM rejects alternatives with value/utility below the lower threshold, and a linear veto function between 0 and 1 is used to reduce the global value/utility of the alternative otherwise.

In ranking problems, the veto function rejects the position of the vetoed alternative in the ranking process rather than the alternative itself. The weighted veto functions of all attributes are aggregated by summation and incorporated into the additive model and the global value/utility is set to 0 if the performance of the alternative is unacceptable for all attributes. Otherwise, the global value/utility of an alternative vetoed in some attributes is reduced.

In contrast with the additive-veto model proposed in de Almeida (2013), veto values provided by DMs in this paper are precise and expressed in terms of alternative performances rather than alternative utilities, which DMs find easier to do, as they 
suggested at meetings held. Moreover, alternatives whose performances are unacceptable (vetoed) for any of the attributes are rejected in our approach irrespective of the value that they take for other attributes. DMs highlighted this issue.

Moreover, the model in de Almeida (2013) is not applied in a group decisionmaking context by contrast with our approach, where all DMs are allowed to provide veto values, but the corresponding veto is effective for only the most important DMs, whereas veto values corresponding to the remaining DMs are partially taken into account by means of the adjust function.

To conclude, the two approaches cannot be compared with respect to the restoration problem under consideration mainly because the proposal in de Almeida (2013) does not account for a group decision-making context.

On the other hand, the proposal by Daher and de Almeida (2012) accounts for a group decision-making context. It was developed to mitigate the compensatory effects of additive aggregation in group decision-making by introducing ranking vetoes based on a reduction factor to penalize conflicting alternatives and reduce disagreements. Specifically, Daher and de Almeida's proposal is based on three concepts: veto thresholds, the virtual alternative and the utility reduction factor $(U R F)$.

Veto threshold, $\alpha_{l}$, represents the minimum acceptable utility value for $D M_{l}$, i.e. any alternative for which the individual utility value is less than $\alpha_{l}$ is considered as unacceptable or undesirable for that DM and potentially conflicting.

Alternative $A_{\alpha}$ is a virtual alternative consisting of all individual utility values in $\alpha=\left(\alpha_{1}, \ldots, \alpha_{k}\right)$. The aggregation of all individual utility values of $\alpha$ defines the global utility of a virtual alternative, $u\left(A_{\alpha}\right)$. When a vector representation is used, the virtual alternative affords four different sub-areas, created by the intersection of threshold values: a positive concordance zone where all DMs are willing to accept alternatives placed in that region; a negative concordance zone where all DMs are willing to reject alternatives placed in that region; and discordance zones where at least one DM considers an alternative placed in that region as unacceptable or undesirable as a group solution.

Finally, the $U R F$ is used to penalize any alternative located in discordance zones, as follows:

$$
u\left(A_{i}\right)=U R F \times \sum_{l=1}^{k} w_{D M_{l}} u^{l}\left(A_{i}\right),
$$

where $U R F=1$, if the alternative belongs to a concordance zone (positive or negative), or $U R F=u\left(A_{\alpha}\right)$, if an alternative belongs to a discordance zone and has an original utility value higher than the virtual alternative utility value.

The utility value of potentially conflicting alternatives is reduced to the same value as the virtual alternative, and, consequently, they have the same rank position. Different $U R F$ rules such as a linear or geometric $U R F$ could be considered.

Note that the approach by Daher and de Almeida does not consider decision-making with partial or imprecise information and the group ranking is derived from an additive form that accounts for the relative importance of DMs, see Eq. (2). However, we account for uncertainty about the alternative performances and imprecision concerning DM preferences, which is exploited together with veto values in a dominance 
measuring method to derive rankings of alternatives, which are then aggregated using the OEA algorithm.

Daher and de Almeida's approach cannot be adapted to the group decision-making context since veto thresholds represent the minimum acceptable utility value for DMs, whereas our rankings are built on the basis of global dominance intensities.

\section{Conclusions}

In this paper we have dealt with a real group decision-making problem centered on the restoration of a radionuclides contaminated lake. We have extended the additive multiattribute utility model to incorporate the concept of veto in this group decisionmaking context. Additionally, we have considered uncertainty about the alternative performances and imprecision concerning DMs' preferences, which can be represented by intervals or ordinal information.

We have proposed a methodology consisting of two aggregation phases. In the first phase, the additive multi-attribute utility model is adapted to account for DM vetoes. Veto values for the most important DMs are used to define veto ranges, whereas veto values corresponding to the other less important DMs are partially taken into account, leading to the construction of adjust ranges. Then, a dominance measuring method, a recent approach for dealing with incomplete information based on the pairwise dominance notion is used to derive a ranking of alternatives for each DM.

In the second phase, the different rankings derived in the first phase are aggregated taking into account the relative importance of DMs to reach a group ranking using the order explicit algorithm method, an extension of Kemeny optimal aggregation.

Acknowledgements The paper was supported by the Spanish Ministry of Economy and Competitiveness MTM2014-56949-C3-2-R.

\section{References}

Aguayo EA (2014) Dominance intensity methods for ranking of alternatives in MCDM with imprecise information. Ph.D. Thesis, Universidad Politécnica de Madrid, Madrid

Aguayo E, Mateos A, Jiménez-Martín A (2014) A new dominance intensity method to deal with ordinal information about a DM's preferences within MAVT. Knowl Based Syst 69:159-169

Ahn BS, Park KS (2008) Comparing methods for multiattribute decision making with ordinal weights. Comput Oper Res 35:1660-1670

Arrow KJ, Raynaud H (1986) Social choice and multicriterion decision making. MIT Press, Cambridge

Bana e Costa CA, Corra E, De Corte JM, Vansnick JC (2002) Faciliting bid evalutation in public call for tenders: a social-technical approach. Omega 30:227-242

Barron F, Barret B (1996) Decision quality using ranked attribute weights. Man Sci 42:1515-1523

Borda J (1981) Memoire sur les elections au scrutin. Histoire de l'Academie des Sciences, Paris

Daher SSD, de Almeida AT (2012) The use of ranking veto concept to mitigate the compensatory effects of additive aggregation in group decisions on a water utility automation investment. Group Decis Negot 21:185-204

de Almeida AT (2013) Additive-veto models for choice and ranking multicriteria decision problems. Asia Pac J Oper Res 30:1350026

DeConde R, Hawley S, Falcon S, Clegg N, Knudsen B, Etzioni R (2006) Combining results of microarray experiments: a rank aggregation approach. Stat Appl Genet Mol Biol 5:5-15 
Ehrhardt J, Brown J, French S, Kelly GN, Mikkelsen T, Müller H (1997) RODOS: decision-making support for off-site emergency management after nuclear accidents. Kerntechnik 62:122-128

Eum Y, Park K, Kim H (2001) Establishing dominance and potential optimality in multi-criteria analysis with imprecise weights and value. Comput Oper Res 28:397-409

Gallego E, Magán M (2009) Preparation, performance and evaluation of the application of the MOIRA DSS to evaluate rehabilitation strategies for contaminated lakes (local scale). Report EURANOS(DEM)TN(09)-08, Universidad Politécnica de Madrid, Madrid

Gallego E, Magán M, Cadierno JPG, Gil E, Monte L, Hofman D (2009) Long-term management of contaminated freshwater bodies and catchments. Decision making exercise with the MOIRA system. Radioprotection 44:683-688

Greco S, Kadzinski M, Mousseau V, Slowinski R (2011) ELECTREGKMS: robust ordinal regression for outranking methods. Eur J Oper Res 214:118-135

Green P (1978) Research for marketing decisions. Prentice-Hall, New Jersey

Håkanson L, Gallego E, Ríos-Insua S (2000) The application of the lake ecosystem index in multi-attribute decision analysis in radioecology. J Environ Radioact 49:319-344

Hwang CL, Yoon K (1981) Multiple attribute decision-making: methods and applications. Springer, Berlin

Jiménez A, Ríos-Insua S, Mateos A (2003) A decision support system for multiattribute utility evaluation based on imprecise assignments. Decis Support Syst 36:65-79

Jiménez A, Mateos A, Ríos-Insua S (2005) Monte Carlo simulation techniques in a decision support system for group decision making. Group Decis Negot 14:109-130

Jiménez A, Mateos A, Sabio P (2013) Dominance intensity measure within fuzzy weight oriented MAUT: an application. Omega 41:397-405

Kemeny J (1959) Mathematics without numbers. Daedalus 88:577-591

Khatib-Rahbar M et al (1998) A regulatory evaluation of the CN Almaraz probabilistic safety analysis (level-2). Report ERI/CSN 98-801

Lahdelma R, Salminen P (2001) SMAA-2: stochastic multicriteria acceptability analysis for group decision making. Oper Res 49:444-454

Lahdelma R, Hokkanen J, Salminen P (1998) SMAA-stochastic multiobjective acceptability analysis. Eur J Oper Res 106:137-1438

Lahdelma R, Miettinen K, Salminen P (2003) Ordinal criteria in stochastic multi-criteria acceptability analysis (SMAA-O). Eur J Oper Res 147:117-127

Lee K, Park K, Kim S (2002) Dominance, potential optimality, imprecise information, and hierarchical structure in multi-criteria analysis. Comput Oper Res 29:1267-1281

Liginlala D, Ow TT (2006) Modeling attitude to risk in human decision processes: an application of fuzzy measures. Fuzzy Sets Syst 157:3040-3054

Lin S (2010) Rank aggregation methods. WIREs Comput Stat 2:555-570

Lin S, Ding J (2009) Integration of ranked lists via cross entropy Monte Carlo with applications to mRNA and microRNA studies. Biometrics 65:9-18

Marichal JL (2004) Tolerant or intolerant character of interacting criteria in aggregation by the Choquet integral. Eur J Oper Res 155:771-791

Mateos A, Jiménez A, Ríos-Insua S (2003) A multiattribute solving dominance and potential optimality in imprecise multiattribute additive problems. Reliab Eng Syst Saf 79:253-262

Mateos A, Ríos-Insua S, Jiménez A (2007) Dominance, potential optimality and alternative ranking in imprecise decision making. J Oper Res Soc 58:326-336

Mateos A, Jiménez-Martín A, AguayoE, Sabio P (2014) Dominance intensity measuring methods in MCDM with ordinal relations regarding weights. Knowl Based Syst 70:26-32

Monte L, Brittain J (eds.) (1998) Principles for the development and implementation of the MOIRA computerised system. Report RT/AMB/98/4, ENEA, Rome

Monte L, van der Steen J, Bergstroem U, Gallego E, Brittain J, Håkanson L (eds.) (2000) The project MOIRA: a model-based computerised system for management support to identify optimal remedial strategies for restoring radionuclide contaminated aquatic ecosystems and drainage areas. Final Report RT/AMB/2000/13 ENEA, Rome

Monte L, Kozhouharov V, Zheleznyak M, Kryshev I, Voitsekhovitch O, Brittain J, Gallego E, Håkanson L (2002) (eds.) Implementing computerised methodologies to evaluate the effectiveness of countermeasures for restoring radionuclide contaminated fresh water ecosystems. COMETES Project. Final Report RT/AMB/2001/2, ENEA, Rome 
Monte L, Hofman D, Brittain J (eds.) (2005) Evaluation and network of EC-decision support systems in the field of hydrological dispersion models and of aquatic radioecological research. EVANET-HYDRA Project. Report RT/2005/49/PROT, ENEA, Rome

Monte L, Brittain J, Gallego E, Håkanson L, Hofman D, Jiménez A (2009) MOIRA-PLUS: a decision support system for the management of complex fresh water ecosystems contaminated by radionuclides and heavy metals. Comput Geosci 35:880-896

Moulin H (1981) The proportional veto principle. Rev Econ Stud 48:407-416

Munda G (2009) A conflict analysis approach for illuminating distributional issues in sustainability. Eur J Oper Res 194:307-322

Nowak M (2004) Preference and veto threshold in multicriteria analysis based on stochastic dominance. Eur J Oper Res 158:339-350

Pihur V, Datta S, Datta S (2009) RankAggreg, an R package for weighted rank aggregation. BMC Bioinform 62:10

Puerto J, Mármol AM, Monroy L, Fernández FR (2000) Decision criteria with partial information. IEEE Trans Oper Res 7:51-65

Raiffa $H$ (1982) The art and science of negotiation. Harvard University Press, Cambridge

Raskob W, Gering F, Lochard J, Nisbet A, Starostova V, Tomic B (2010) Overview and main achievements of the EURANOS project: European approach to nuclear and radiological emergency management and rehabilitation strategies. Radioprotection 45:S9-S22

Ríos Insua D (1990) Sensitivity analysis in multi-objective decision making. Springer, New York

Ríos D, French S (1991) A framework for sensitivity analysis in discrete multiobjective decision-making. Eur J Oper Res 54:176-190

Roy B, Slowinski R (2008) Handling effects of reinforced preference and counter-veto in credibility of outranking. Eur J Oper Res 188:185-190

Sabio P, Jiménez-Martín A, Mateos A (2015) Veto values within MAUT for group decision making on the basis of dominance measuring methods with fuzzy weights. LNBIP 218:1-12

Salo A, Hämäläinen RP (2001) Preference ratio in multiattribute evaluation (PRIME)-elicitation and decision procedures under incomplete information. IEEE Trans Syst Man Cybern Part A 31:533-545

Sarabando P, Dias LC (2009) Multi-attribute choice with ordinal information: a comparison of different decision rules. IEEE Trans Syst Man Cybern Part A 39:545-554

Sarabando P, Dias LC (2010) Simple procedures of choice in multicriteria problems without precise information about the alternatives values. Comput Oper Res 37:2239-2247

Stewart T (1996) Robustness of additive value function method in MCDM. J Multicriteria Decis Anal 5:301-309

Stillwell WG, Seaver DA, Edwards WA (1981) Comparison of weight approximation techniques in multiattribute utility decision making. Org Behav Hum Decis Proc 28:62-77

Vetschera R (1990) Group decision and negotiation support—a methodological survey. OR Spektrum 12:67_ 77

Weber M (1987) Decision making with incomplete information. Eur J Oper Res 28:44-57

Yoon K (1980) System selection by multiple attribute decision making. Ph.D. Thesis, Kansas State University Press, Manhattan

Yue Z (2011) A method for group decision-making based on determining weights of decision makers using TOPSIS. Appl Math Model 35:1926-1936 